\title{
Planned Four-stage Transforaminal Full-endoscopic Lumbar Decompression under Local Anesthesia in a Patient with Severe Comorbidity
}

\author{
Ayaka HASHIMOTO, ${ }^{1}$ Fumitake TEZUKA, ${ }^{1}$ Kazuta YAMASHITA, ${ }^{1}$ \\ Masatoshi MORIMOTO, ${ }^{1}$ Kosuke SUGIURA, ${ }^{1}$ Makoto TAKEUCHI, ${ }^{1}$ \\ Yoichiro TAKATA, ${ }^{1}$ Toshinori SAKAI, ${ }^{1}$ Toru MAEDA, ${ }^{1}$ and Koichi SAIRYO ${ }^{1}$ \\ ${ }^{1}$ Department of Orthopedics, Tokushima University, Tokushima, Tokushima, Japan
}

\begin{abstract}
A 74-year-old man presented with symptoms of intermittent claudication. A diagnosis of lumbar spinal canal stenosis (LSS) at L3/4 and L4/5 was made based on the clinical and radiological findings. Bilateral lateral recess stenosis was detected at both these levels on magnetic resonance imaging (MRI) and on computed tomography (CT) scans obtained after myelography. Four nerve roots were impinged bilaterally at L4 and L5. The initial plan was to perform conventional laminectomy at L3/4 and L4/5 under general anesthesia. However, the neurologists pointed out that the patient had comorbidities of parkinsonism and severe carotid artery stenosis, meaning that an increase or decrease in blood pressure during general anesthesia could cause a stroke. Therefore, we changed the surgical plan to four-stage full-endoscopic ventral facetectomy at L3/4 and L4/5 bilaterally under local anesthesia. There were no surgery-related complications after any of the four operations. The patient's symptoms improved after the final operation and the modified MacNab criteria indicated a good clinical outcome. Full-endoscopic lumbar decompression surgery under local anesthesia may be effective in elderly patients who are in poor general health.
\end{abstract}

Keywords: transforaminal full-endoscopic lumbar decompression, lateral recess stenosis, ventral facetectomy, local anesthesia

\section{Introduction}

Full-endoscopic spine surgery (FESS) requires only an $8-\mathrm{mm}$ skin incision and causes minimal damage to the back muscles. Therefore, it is suitable for professional athletes ${ }^{1)}$ and allows a rapid return to work postoperatively. ${ }^{2,3)}$ FESS may be performed via a transforaminal (TF) or interlaminar approach. ${ }^{4)}$ In general, interlaminar FESS requires general anesthesia, whereas TF-FESS can be performed under local anesthesia. ${ }^{5)}$ A procedure that can be performed under local anesthesia is desirable in elderly patients, especially those in poor general health.

TF-FESS was first used for lumbar discectomy 20 years ago. ${ }^{6-8)}$ Since then, an ultra-thin high-speed

Received April 11, 2020; Accepted September 7, 2020

Copyright $\subseteq 2021$ by The Japan Neurosurgical Society This work is licensed under a Creative Commons AttributionNonCommercial-NoDerivatives International License. surgical drill that can be used in a small working space had become available, ${ }^{9,10)}$ allowing surgeons to perform decompression for spinal canal stenosis by FESS. Initially, foraminal stenosis was decompressed by full-endoscopic lumbar foraminotomy (FELF) using a TF full-endoscopic system. ${ }^{11,12)}$ More recently, it has become possible to decompress lateral recess stenosis via a TF approach ${ }^{3,5,13)}$ known as TF full-endoscopic ventral facetectomy (FEVF). Both TF-FELF and TF-FEVF can be performed under local anesthesia, meaning that patients who are unsuitable for general anesthesia can undergo these procedures.

Here we report the case of an elderly patient in poor general health in whom we performed successful four-stage FEVF for bilateral lateral recess stenosis at $\mathrm{L} 3 / 4$ and $\mathrm{L} 4 / 5$.

\section{Case Report}

A 74-year-old man who had been treated for Parkinson's disease at our neurology department was 

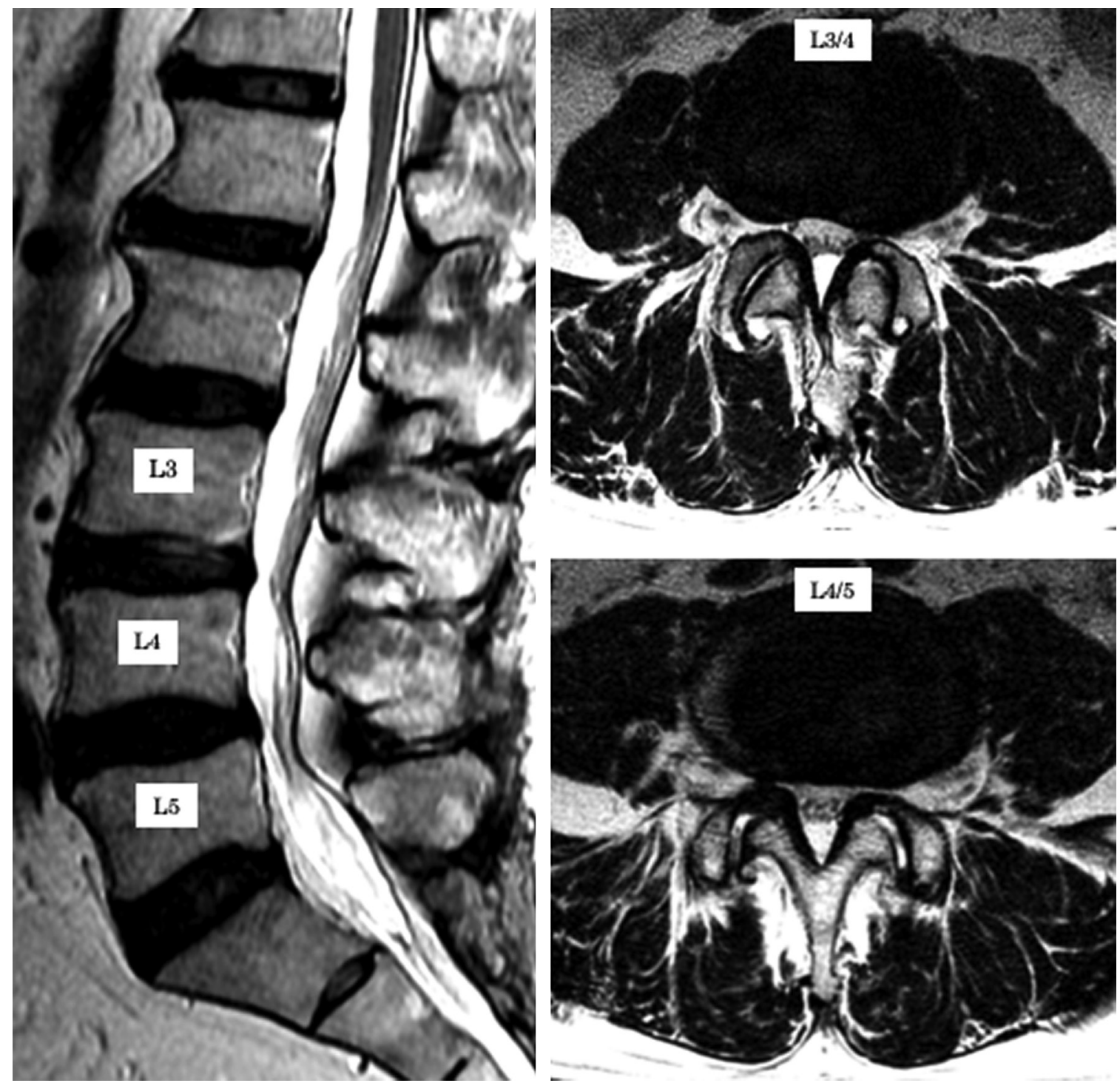

Fig. 1 Magnetic resonance scans acquired at the initial presentation. Bilateral lateral recess stenosis is evident at L3/4 and L4/5 on axial images. No central stenosis is seen on a sagittal image.

referred to us with a history of both leg pain and neurogenic intermittent claudication for several years. He was using a wheelchair to mobilize outside his home because of his leg pain at L4 and L5. There was no muscle weakness or sensory disturbance in either leg. His Achilles and patellar tendon reflexes were essentially normal. Tension signs, including the femoral nerve stretch and straight leg raise tests, were negative.

Magnetic resonance scans at the initial presentation revealed bilateral lateral recess stenosis at L3/4 and L4/5 on axial images with no central stenosis seen on a sagittal image (Fig. 1). The findings on computed tomography (CT) scans obtained after myelography were similar to those on the magnetic resonance imaging (MRI). All four nerve roots at
L4 and L5 were impinged by lateral recess stenosis (Fig. 2). Therefore, we initially planned to perform traditional laminectomy at $\mathrm{L} 3 / 4$ and $\mathrm{L} 4 / 5$ under general anesthesia to treat this pathology.

His preoperative sonographic characteristics of bilateral carotid arteries showed that there was isoto high-echoic plaques and calcification in bilateral carotid arteries, the unstable plaque with lipid rich in the left common carotid sinus. Mean intima media thickness (IMTs) were $0.91 \mathrm{~mm}$ in both sides and the total plaque score was $22 \mathrm{~mm}$ (right: $11.5 \mathrm{~mm}$, left $10.5 \mathrm{~mm}$ ). Peak systemic velocities of the common carotid artery (CCA) were $75 \mathrm{~cm} / \mathrm{s}$ on the right side and $89 \mathrm{~cm} / \mathrm{s}$ on the left side. End diastolic velocities (EDV) of CCA were $12 \mathrm{~cm} / \mathrm{s}$ in the right side and $19 \mathrm{~cm} / \mathrm{s}$ in the left side. There was no significant 

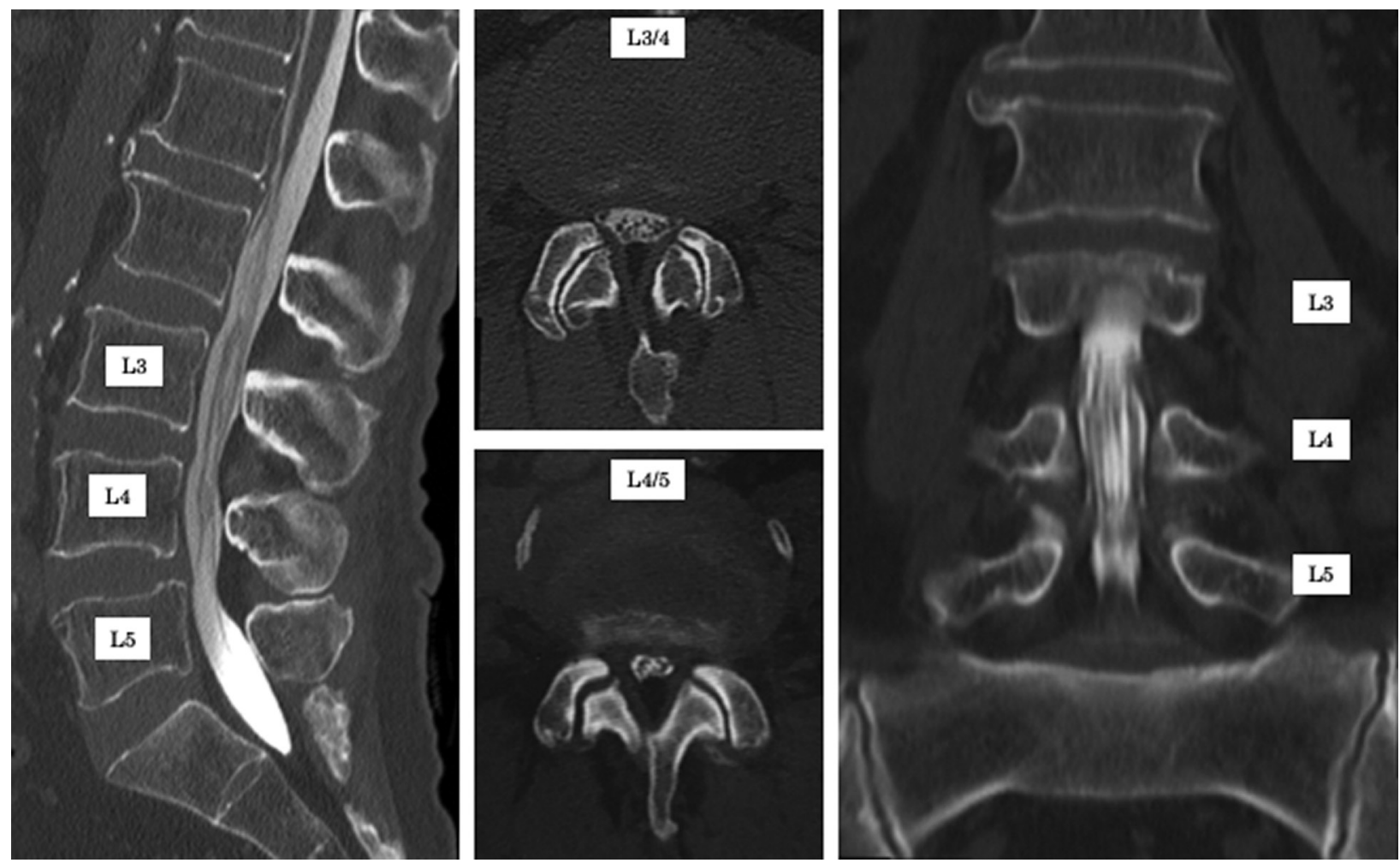

Fig. 2 CT scans obtained after myelography before surgery. Four nerve roots (at L4 and L5 bilaterally) are impinged by lateral recess stenosis. CT: computed tomography.

stenosis in carotid arteries within the sonographic observation; however, compared to the left side, EDV in right CCA was decreased, which suspected that the peripheral side of the right internal carotid artery might be stenotic. Cerebral magnetic resonance angiography showed asymptomatic internal carotid artery stenosis on the right side. He has never had a past medical history of cerebral infarction, oral medication of antiplatelet drugs, or statins. The neurologist and anesthesiologist concluded that he could have high risk of cerebral infarction as a result of a sudden change in blood pressure during general anesthesia. Moreover, he had past medical history of chronic obstructive pulmonary disease (COPD) with decreased forced expiratory volume of $59.0 \%$. Therefore, we changed the surgical plan to four-stage FEVF bilaterally at L3/4 and L4/5 under local anesthesia.

An FEVF procedure is performed under local anesthesia with stabilization of blood pressure and adequate pain control and sedation by an anesthesiologist. After creating an 8-mm skin incision about 7-8 cm from the midline, local anesthesia is injected into the subcutaneous tissue, fascia, paravertebral muscle tissue, and facet joint. First, an 8-mm cannula is docked on the superior articular process. The entire superior articular process is then removed using a high-speed drill. Next, the inferior articular process is partially removed, exposing the ligamentum flavum in the area that covers the traversing nerve root. Finally, the ligamentum flavum at the lateral recess is resected, and ventral facetectomy is completed after confirmation that the traversing nerve is decompressed.

In our patient, FEVF was performed first at L4/5 on the right side and then on the left side 2 months later. At that time, the patient's lower leg pain resolved completely but he continued to experience anterior thigh pain when walking. Therefore, we planned FEVF at L3/4. Unfortunately, the patient developed pneumonia just before surgery. Therefore, the operation was deferred until he had recovered. Two years after FEVF at L4/5, when his general condition had improved, FEVF was performed at L3/4 on the right side and then at L3/4 on the left side 1 month later. No surgery-related complications (such as nerve root damage, dural tearing, hematoma, or infection) were recorded after any of the four operations.

Postoperative CT scans confirmed successful ventral facetectomy and bony resection at both L3/4 and L4/5, and MRI showed decompression of the traversing nerve roots of both L4 and L5 (Fig. 3). Comparison of three-dimensional CT scans obtained before and after the surgery (Fig. 4) confirmed that 

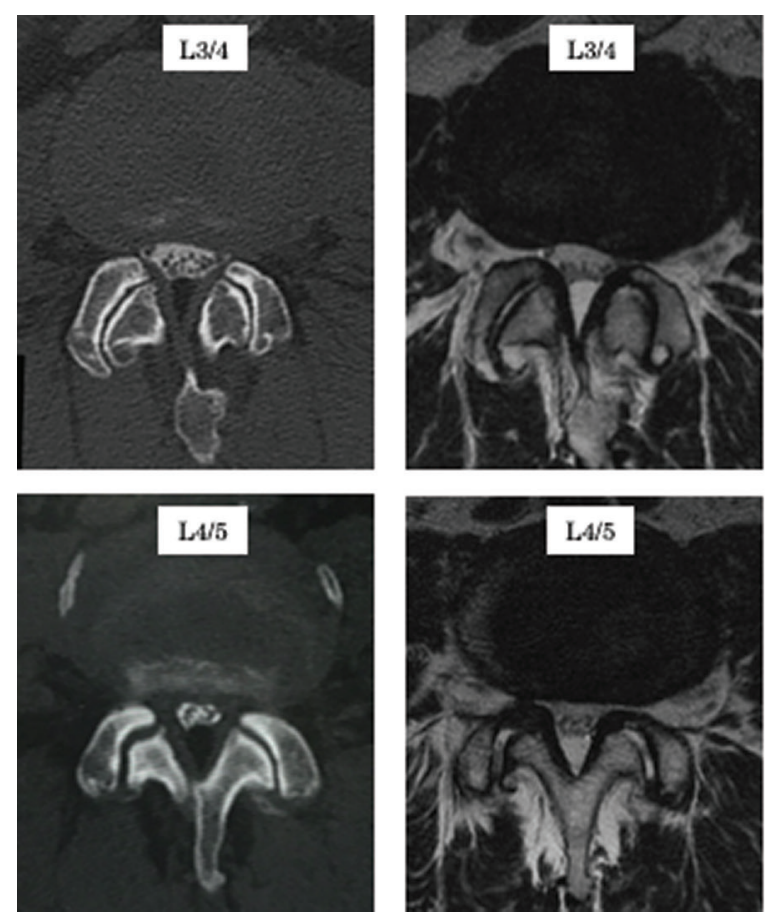

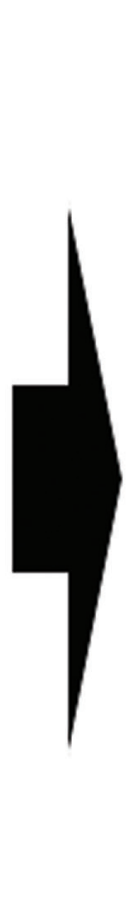

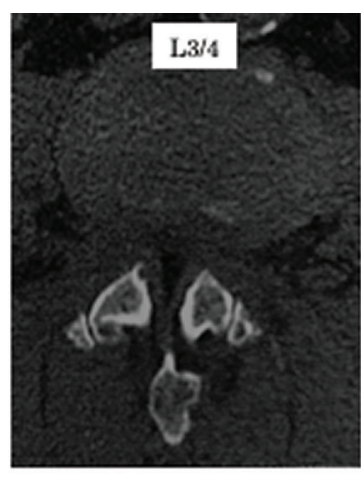
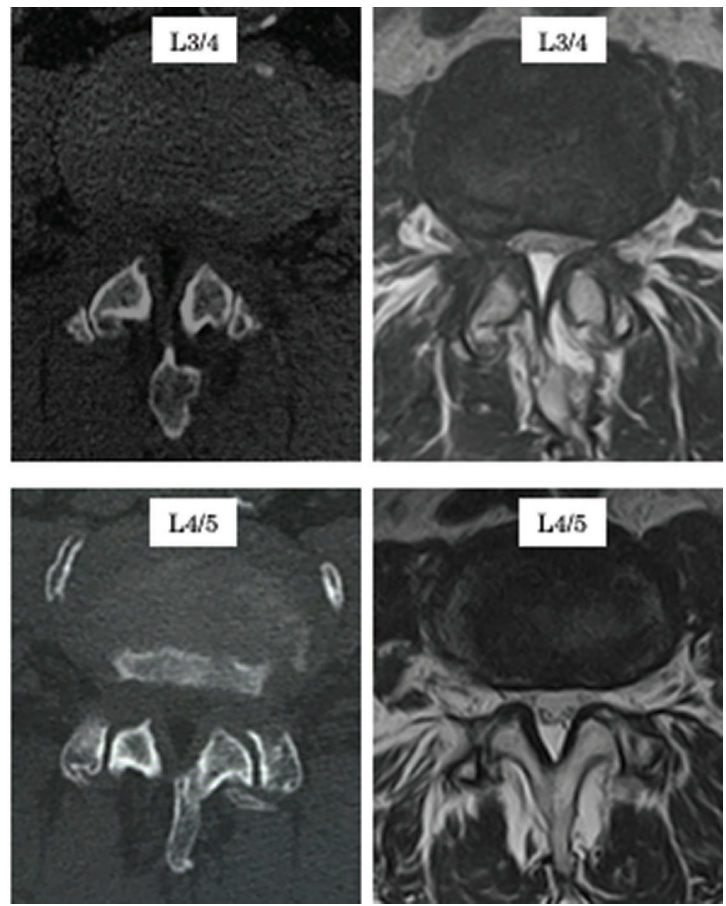

Fig. 3 Preoperative and postoperative CT scans and MRI. Successful ventral facetectomy with bony resection is confirmed at the L3/4 and L4/5 levels after the four surgical procedures. CT: computed tomography, MRI: magnetic resonance imaging.

bony resection performed through the intervertebral foramen was adequate at L3/4 and L4/5. During follow-up period, we did not confirm segmental instability at L3/4 and L4/5 after the operations.

The postoperative course was good after the final surgery. The pain in both legs was improved and the patient could walk without intermittent claudication. According to the modified MacNab criteria, the surgical outcome is good.

\section{Discussion}

In this report, we have described a man who presented with lumbar spinal canal stenosis (LSS) at L3/4 and L4/5 but had surgical risk factors of parkinsonism, severe carotid artery stenosis, and COPD. Therefore, there was a substantial risk of stroke as a result of change in blood pressure during general anesthesia. We elected to perform TF-FEVF under local anesthesia in this patient. His pathology was bilateral lateral recess stenosis at L3/4 and L4/5; therefore, a total of four nerve roots, including those at L4 and L5 bilaterally, were impinged by the stenosis. Four-stage TF-FEVF ${ }^{3,5,13)}$ at L3/4 and L4/5 was performed stepby-step for this pathology. There were no surgery-related complications and his symptoms are now alleviated.

LSS is the most common spinal disorder in the elderly population. The gold standard surgical treatment for LSS is laminectomy. Although The invasiveness of laminectomy is now minimal when using a microscope, ${ }^{14)}$ microendoscope, ${ }^{15)}$ or fullendoscope, ${ }^{16)}$ interlaminar procedures still require general anesthesia. Although $\mathrm{Wu}$, et al. reported stepwise local anesthesia for percutaneous endoscopic discectomy via interlaminar approach, ${ }^{17)}$ this procedure is typically performed under general anesthesia. Full-endoscopic TF procedure can be performed under local anesthesia. Many elderly patients with LSS have poor general health, with comorbidities such as cardiac or pulmonary dysfunction, which typically renders general anesthesia impossible. TF full-endoscopic decompression is worthwhile in such cases because it can be performed under local anesthesia. Of course, local anesthesia has potential risks of respiratory depression or hemodynamic instability due to the use of sedative drugs and analgesics as well. Therefore, we should pay attention to monitor patient's vital signs during operation. In our case, four FEVFs could be performed under local anesthesia with help of an anesthesiologist.

Transforaminal full-endoscopic lumbar discectomy (TELD) through Kambin's safety triangle is one of the posterolateral approaches and can be performed with $8 \mathrm{~mm}$ skin incision and minimal disruption of the spinal structures including ligaments and muscles. 

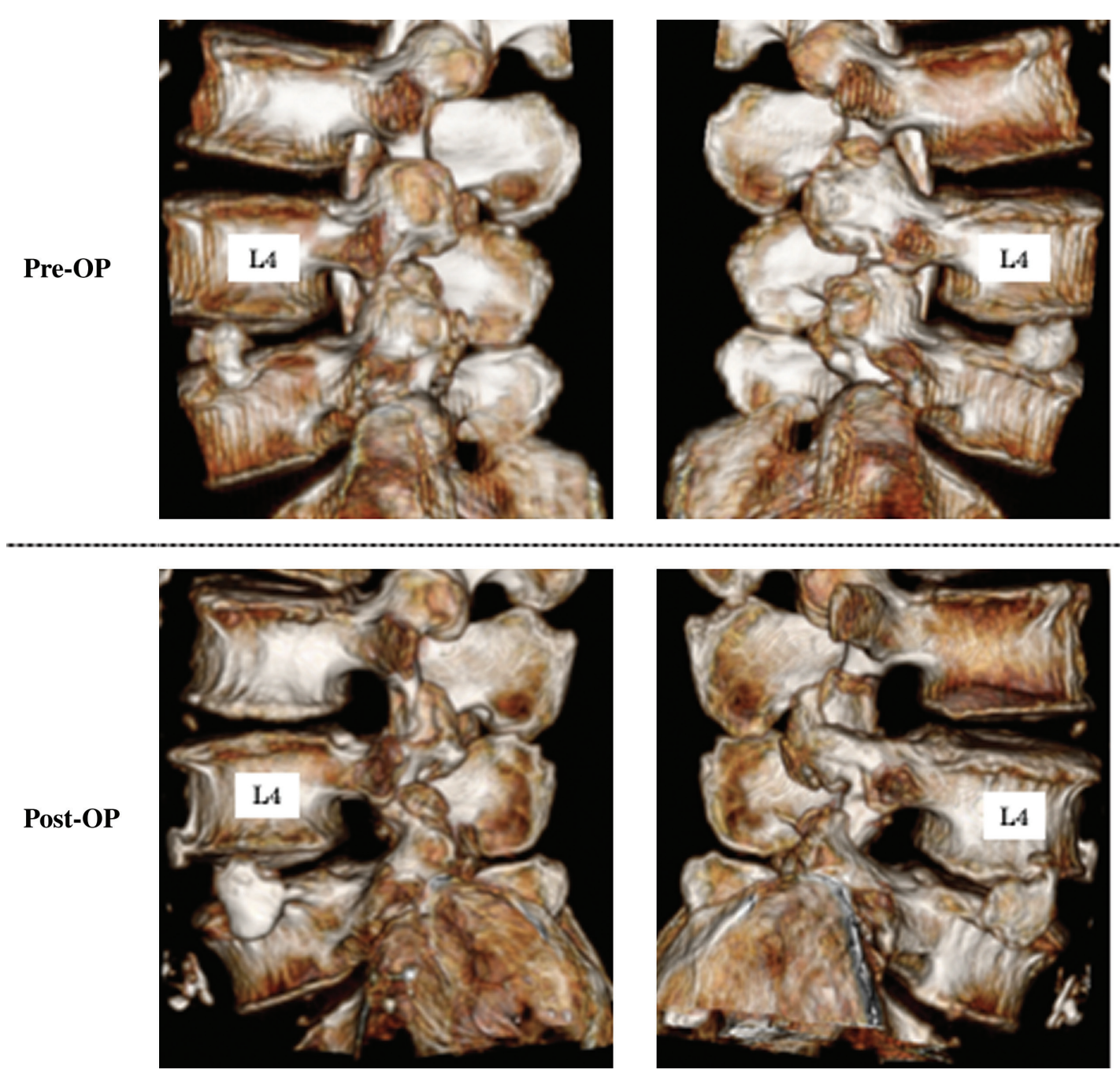

Fig. 4 Three-dimensional CT scans obtained before and after the surgery. Bony resection performed through the intervertebral foramen is adequate at L3/4 and L4/5. CT: computed tomography.

According to the result of a meta-analysis compared TELD compared to standard discectomy for the treatment of lumbar disc herniation (LDH), TELD found to be superior to those after standard discectomy for blood loss, operative time, hospital stay, and mean disability period. ${ }^{18)}$ Postoperative complications of $\mathrm{TF}$ procedures are dysesthesia in exiting nerve root distribution, nerve root injury, and revision operation regarding to incomplete decompression. Incidence of them is reported $3.1-9.1 \% .^{19,20)}$ There are no report of postoperative juxta-facet cyst regarding to TF-FESS. In our case, preoperative MRI showed fluid collection in both facet joints at L4/5, which suggested segmental instability of L4/5 and potential risk of juxta-facet cyst after this operation. Although this patient has never had juxta-facet cyst, we should follow up in the long-term period.

This case states the usefulness of FEVF in that it can be performed under local anesthesia for elderly patients in poor general health. Another advantage of this procedure is that it is minimally invasive. The skin incision is only $8 \mathrm{~mm}$ and muscle damage is minimal, meaning that the patient can resume daily activities very rapidly. For example, Sugiura et al. ${ }^{3}$ reported a case of a male dentist with bilateral L4/5 lateral recess stenosis who returned to work 5 days after FEVF. Fujii et al. ${ }^{2}$ reported similar results in physician patients, one of whom underwent FEVF and returned to work just 2 days after surgery. Therefore, TF full-endoscopic decompression can be considered effective not only in elderly patients with comorbidities but also in patients who need a rapid return to work after surgery.

The number of patients undergoing LSS is likely to be increasing in developed countries, all of which are experiencing a steady annual increase in their elderly populations. The elderly are very likely to 
have comorbidities, such as hypertension and diabetes; if severe, these comorbidities preclude surgery under general anesthesia. For example, Sairyo et al. ${ }^{21)}$ reported the case of a 72-year-old woman with central LSS and severe pulmonary dysfunction (minute volume, $0.71 \mathrm{~L}$ ). In this patient, they performed a novel TF full-endoscopic procedure under local anesthesia to decompress the central stenosis, including total resection of the superior articular process, partial removal of the inferior articular process, and partial flavectomy, which they referred to as TF lumbar undercutting laminectomy.

There are three types of LSS, that is, foraminal, lateral recess, and central. TF full-endoscopic decompression was first developed for foraminal stenosis. ${ }^{11,12)}$ Subsequently, it was found that lateral recess stenosis could be decompressed using a TF approach. ${ }^{3,5,13)}$ Finally, it became possible to decompress central stenosis by $\mathrm{TF}$ lumbar undercutting laminectomy under local anesthesia. ${ }^{21)}$ There are some ongoing technical limitations, but we are now able to decompress any type of LSS under local anesthesia using a TF full-endoscopic system.

\section{Conclusion}

We performed four-stage TF FEVF at L3/4 and L4/5 bilaterally in step-by-step manner under local anesthesia in a man with LSS at L3/4 and L4/5 who had severe comorbidities. The TF full-endoscopic decompression under local anesthesia can be recommended for elderly patients with a poor general health status.

\section{Conflicts of Interest Disclosure}

The authors have no conflict of interest concerning the materials or methods used in this study or the findings specified in this paper.

\section{References}

1) Maeda T, Takamatsu N, Hashimoto A, et al.: Return to play in professional baseball players following transforaminal endoscopic decompressive spine surgery under local anesthesia. J Spine Surg 6: S300-S306, 2020

2) Fujii Y, Yamashita K, Sugiura K, et al.: Early return to activity after minimally invasive full endoscopic decompression surgery in medical doctors. J Spine Surg 6: S294-S299, 2020

3) Sugiura K, Yamashita K, Manabe H, et al.: Prompt return to work after bilateral transforaminal fullendoscopic lateral recess decompression under local anesthesia: a case report. J Neurol Surg A Cent Eur Neurosurg doi: 10.1055/s-0040-1712463 Epub 2020 Dec 22
4) Ruetten S, Komp M, Merk H, Godolias G: Fullendoscopic interlaminar and transforaminal lumbar discectomy versus conventional microsurgical technique: a prospective, randomized, controlled study. Spine (Phila Pa 1976) 33: 931-939, 2008

5) Sairyo K, Chikawa T, Nagamachi A: State-ofthe-art transforaminal percutaneous endoscopic lumbar surgery under local anesthesia: Discectomy, foraminoplasty, and ventral facetectomy. J Orthop Sci 23: 229-236, 2018

6) Yeung AT: The evolution of percutaneous spinal endoscopy and discectomy: state of the art. $M t$ Sinai J Med 67: 327-332, 2000

7) Yeung AT, Tsou PM: Posterolateral endoscopic excision for lumbar disc herniation: Surgical technique, outcome, and complications in 307 consecutive cases. Spine (Phila Pa 1976) 27: 722-731, 2002

8) Sairyo K, Nagamachi A: State-of-the-art management of low back pain in athletes: instructional lecture. J Orthop Sci 21: 263-272, 2016

9) Dezawa A, Sairyo K: New minimally invasive discectomy technique through the interlaminar space using a percutaneous endoscope. Asian J Endosc Surg 4: 94-98, 2011

10) Dezawa A, Mikami H, Sairyo K: Percutaneous endoscopic translaminar approach for herniated nucleus pulposus in the hidden zone of the lumbar spine. Asian J Endosc Surg 5: 200-203, 2011

11) Yamashita K, Higashino K, Sakai T, et al.: Percutaneous full endoscopic lumbar foraminoplasty for adjacent level foraminal stenosis following vertebral intersegmental fusion in an awake and aware patient under local anesthesia: a case report. J Med Invest 64: 291-295, 2017

12) Yeung A, Gore S: Endoscopic foraminal decompression for failed back surgery syndrome under local anesthesia. Int J Spine Surg 8: 22, 2014

13) Sairyo K, Higashino K, Yamashita K, et al.: A new concept of transforaminal ventral facetectomy including simultaneous decompression of foraminal and lateral recess stenosis: Technical considerations in a fresh cadaver model and a literature review. J Med Invest 64: 1-6, 2017

14) McCulloch JA, Snook D, Kruse CF: Advantages of the operating microscope in lumbar spine surgery. Instr Course Lect 51: 243-245, 2002

15) Wada K, Sairyo K, Sakai T, Yasui N: Minimally invasive endoscopic bilateral decompression with a unilateral approach (endo-BiDUA) for elderly patients with lumbar spinal canal stenosis. Minim Invasive Neurosurg 53: 65-68, 2010

16) Ruetten S, Komp M, Hahn P, Oezdemir S: Decompression of lumbar lateral spinal stenosis: full-endoscopic, interlaminar technique. Oper Orthop Traumatol 25: 31-46, 2013

17) Wu K, Zhao Y, Feng Z, Hu X, Chen Z, Wang Y: Stepwise local anesthesia for percutaneous endoscopic interlaminar discectomy: technique strategy and clinical outcomes. World Neurosurg 134: e346-e352, 2020 
18) Li X, Han Y, Di Z, et al.: Percutaneous endoscopic lumbar discectomy for lumbar disc herniation. J Clin Neurosci 33: 19-27, 2016

19) Kapetanakis S, Gkantsinikoudis N, Thomaidis T, Charitoudis G, Theodosiadis P: The role of percutaneous transforaminal endoscopic surgery in lateral recess stenosis in elderly patients. Asian Spine J13: 638-647, 2019

20) Li Y, Wang B, Wang S, Li P, Jiang B: Full-endoscopic decompression for lumbar lateral recess stenosis via an interlaminar approach versus a transforaminal approach. World Neurosurg 128: e632-e638, 2019
21) Sairyo K, Yamashita K, Manabe H, et al.: A novel surgical concept of transforaminal full-endoscopic lumbar undercutting laminectomy (TE-LUL) for central canal stenosis of the lumbar spine with local anesthesia: a case report and literature review. J Med Invest 66: 224-229, 2019

Corresponding author: Koichi Sairyo, MD, PhD Department of Orthopedics, Tokushima University Graduate School, 3-18-15 Kuramoto, Tokushima, Tokushima 770-8503, Japan. e-mail: sairyokun@gmail.com 